\title{
Zum 75. Geburtstag des fast vergessenen Schriftstellers und Justizkritikers
}

Einer unter den zahllosen Vergessenen, die Anrecht gewonnen haben, früher oder später wieder aus dem Dunkel ins Licht der Erinnerung gerufen zu werden, ist der pommersche Pastorensohn Ernst Gottwalt Nicolas, seinerzeit bekannt unter seinem Schriftstellernamen Ernst Ottwalt. Er hat den ganzen Weg durchmessen: vom nationalistischen deutschen Freikorpsmann in der Nachkriegszeit des Ersten Weltkrieges zum überzeugten und engagierten Kommunisten, der dann in den Lagern Stalins endete. Er steht hier stellvertretend für alle, für die es keine Rückkehr mehr gab, und für alle, deren Namen und deren Schicksal im großen Kraftakt der Verdrängung unserer jüngsten Vergangenheit von der einen Seite vergessen wurde, weil er einmal Kommunist war, und der von der anderen Seite geflissentlich totgeschwiegen wird, weil er zu jenen aufrechten Kommunisten gehörte, denen es bestimmt war, zu Opfern des Stalinismus zu werden.

Einmal noch, Jahre nach seinem Tode in einem Lager am Eismeer, wurde sein Wort in der ganzen Welt vernehmlich - durch den Hauptankläger der Sowjetunion bei den Nürnberger Prozessen, Rudenko, und diese Paradoxie gehört mitten ins Bild einer bis zur äußersten Ubersteigerung verworrenen Epoche, in der sich Unrecht von gestern mit Unrecht von heute unauflöslich verschränkt. So wurde Ernst Ottwalt als Kronzeuge der Anklage aufgerufen, obwohl er bereits zum Opfer derer geworden war, die das Unrecht anderer anklagten.

Ernst Ottwalts Frau, Waltraut, kraft eigenen Rechts als Schriftstellerin wie als Mensch Mitzeugin, hat in einem ihrer unter dem Namen Waltraut Nicolas veröffentlichten Bücher, mit dem Titel „Viele tausend Tage», dieser Szene, da vor dem Welttribunal in Nürnberg die Stimme Ottwalts aus dem Grabe nachtönte, in ihrer bizarren Komplexität anschaulich gemacht. Wir geben ihr, die in Deutschland und in der Sowjetunion das Schicksal ihres Mannes teilte, das Wort - auch ihre Stimme schallt nun aus dem Grabe; sie ist 1962 nach sehr leidvollem Leben gestorben:

"... Und nun verlas der sowjetische Ankläger Sätze aus dem Buch, nannte die Seitenzahl, nannte den Buchtitel und den Namen des Autors: "Deutschland erwache, Geschichte des Nationalsozialismus « von Ernst Ottwalt.' Ich wollte es nicht glauben, als ich es in der Zeitung las. Aber da stand es mit aller Deutlichkeit; der Vertreter der Sowjetmacht berief sich in seiner Anklage gegen den Nationalsozialismus einzig auf Ernst, den die gleiche Sowjetmacht als »Feind « in die Arktis verbannt hatte.

Noch am gleichen Tag schrieb ich ein Gesuch an den Bürger Generalleutnant P. A. Rudenko, sowjetischen Hauptankläger beim internationalen Gericht in Nürnberg. Ich schrieb es ganz sachlich, ohne Beschwerde über geschehenes Unrecht, ohne Verteidigung des unschuldig Verurteilten - denn diese Verteidigung hatte ja der Adressat selbst schon besorgt in seiner Rede. Ich machte ihn nur auf ein kleines Versehen aufmerksam, das ihm da unterlaufen war. Ich klärte ihn auf über die Identität des politischen Gefangenen im Lager Archangelsk mit dem Autor des ersten Buches, das gegen den Nationalsozialismus geschrieben worden war und nun

\footnotetext{
1 Wien 1932; fotomechanıscher Nachdruck 1975 durch: Verlag europäische ıdeen, I Berlin 37, Mühlenstr. 17b, 400 S. 30,- DM. Im gleıchen Verlag 1st 1976 erschienen: Ennst Ottwalt, Schriften (Hrsg.: Andreas W Mytze), 258 S. 30,- DM. Demnächst erscheınt Im Verlag europäische ideen: Andreas W Mytze, Ottwalt - Leben und Werk des vergessenen revolutıonären deutschen Schriftstellers (Red. KJ).
} 
dem Nürnberger Hauptankläger als Beweisstück diente. Ich vertraute darauf, schrieb ich, daß er selbst sich für eine gerechte Prüfung des Falles Ernst Ottwalt einsetzen werde. Und ich bat ihn, mittels seines Einflusses meinem Mann die Heimkehr zu ermöglichen - oder mir eine Nachricht zukommen zu lassen, wenn er nicht mehr am Leben sei...

Eine Antwort kam nie. Augenscheinlich machte es der Macht, die über diesen Appell an die Gerechtigkeit zu entscheiden hatte, nichts aus, einen als schuldig verdammten Sträfling gleichzeitig als Propheten zu loben, der einem verblendeten Volk sein Unheil vorausgesagt hatte: bitte sehr, schwarz auf weiß, Seite $353-$ unwiderleglicher Wahrheitszeuge der Anklagebehörde. Heute wurde er gebraucht, seine Stimme als Fanfare des Prozesses; mochte er morgen wieder verstoßen werden in das Schweigen des Verleumdetseins, die Verdammnis der Geächteten ...

Ernst Ottwalt wurde am 13. rr. 1901 in Zippnow, Kreis Deutsch-Krohne (Pommern) geboren. Die Jugend verlebte er in Halle. Von der Schulbank weg reiht sich der natürlich in patriotischem Geiste erzogene Primaner in das "Freikorps Halle" ein, um den »Umsturz « zu bekämpfen, für »Ruhe und Ordnung " zu sorgen, unter dem Schlagwort "Bürger erwache» die "Latjer» wieder in ihre hergebrachten Schranken zurückzuprügeln. Die "Latjer" - damit sind für diese bürgerlichen Gymnasiasten des Kaiserreiches die Arbeiter bezeichnet. Ottwalt erläutert in seinem autobiographischen Roman "Ruhe und Ordnung ${ }^{2}$ : "Latjer, das ist alles, was eine Ballonmütze und keinen Kragen trägt, jenes gestaltlose und vielgestaltige feindliche Etwas, das uns bösartig aus tausend Augen anstiert, wenn wir lärmend und lachend, die weiße Mütze auf dem Ohr und die Zigarette im Mund, bei Schichtwechsel durch die Straßen gehen."

Bald aber bemerkt der Jüngling, daß die Klischees, die ihm anerzogen wurden, unstimmig sind. Er entdeckt mit Ủberraschung, daß »auch Demokraten anständige Menschen sein können«, und diese Entdeckung macht ihn »ratloser als je zuvor «. Während des Kapp-Putsches und der darauf folgenden Kämpfe im mitteldeutschen Gebiet, Halle, Erfurt, Leipzig, wird der Freikorpssoldat beordert, Arbeiterversammlungen zu besuchen, um die Stimmung zu erkunden, womöglich revolutionäre Pläne auszukundschaften - Spitzeldienste, Ottwalt sagt es selbst in dem Kapitel »Der Spitzel«, zu denen sich der noch nicht zwanzigjährige Schüler im Namen des "Vaterlandes« bereit findet; auch Abenteurerlust ist im Spiel. Wichtigtuerei, nicht zuletzt der hohe Sold. Diese nähere Begegnung mit den »Latjern « wird für seine Wandlung bestimmend. In einer persönlichen Mitteilung schrieb er später:

»Es war für mich eine erschütternde und im höchsten Maße beunruhigende Entdekkung, daß die Arbeiter sich den Anteil an den Bildungsgütern des Bürgertums, den die Schule ihnen vorenthielt, selbständig und in erbitterter Arbeit errangen... Lange habe ich mich gegen diese Gefühle und Erkenntnisse gesträubt. Ich versuchte qualvoll, mein ins Nichts zerfließendes und zerbrechendes Weltbild zusammenzuhalten. Ich flüchtete mich in den Überschwang menschenfreundlicher Gefühle, schloß mich der pazifistischen Bewegung an, versuchte dieses und jenes - aber mein Leben blieb leer und inhaltlslos. Ich wurde Redakteur an farblosen Zeitungen, schrieb Berichte und Novellen, kultivierte bacchantisches Bohémetum und rutschte mehr als einmal haarscharf an der moralischen Verlumpung eines absoluten Nihilismus vorbei...《

Für ihn wie für nicht wenige seiner Altersgenossen aus bürgerlichem Haus, darunter manche, die von der nationalen Rechten, dem Freikorps, auch der Hitlerjugend

2 Malik Verlag, Berlin 1929; Nachdruck 1972 durch Paco-Press, Amsterdam. (Red. KJ) 
herkamen, wurde dann der Ausweg aus allen Zweifeln, allen Bedenken die geistige Verankerung im Marxismus und die organisatorische Bindung an die Kommunistische Partei. In ihr glaubte Ottwalt die geistige und soziale Heimat gefunden zu haben, in ihr die Vertretung gerechter Forderungen der von ihm nun nicht verächtlich als "Latjer « bezeichneten, sondern als Lehrmeister empfundenen Arbeiterschaft zu erkennen. Er hat dieser Empfindung, wie es so oft nach vollzogener Bekehrung $\mathrm{zu}$ neuem Glauben oder neuer Weltanschauung geschieht, in schwärmerischen Worten, die uns heute allzu naiv anmuten, aber damals gewiß aus innerer Úberzeugung kamen, Ausdruck gegeben:

"So ist meine schriftstellerische Arbeit für mich nichts als der Wunsch, zu meinem Teil die kämpfende Arbeiterklasse zu unterstützen. Und ich werde glücklich sein, wenn einmal über mein Leben und mein literarisches Schaffen von einem deutschen Arbeiter nichts weiter zu sagen sein wird als dies: er war ein guter Genosse ... « Das literarische Zeugnis dieser Wandlung ist sein 1929 zuerst im Malik-Verlag, Berlin, erschienener Roman »Ruhe und Ordnung «. Er hat dem Buch den Untertitel gegeben: „Roman aus dem Leben der nationalgesinnten Jugend «. In seiner Vorbemerkung sagt er, diese Aufzeichnungen seien ein "wahrheitsgetreues Protokoll eigener Erlebnisse; keine Seite beruht auf freier Erfindung «. Die Romanform habe er gewählt, weil »nicht Schuld oder Verhängnis bestimmter Einzelpersonen dargestellt werden soll, sondern das Bild jener Nachkriegsjugend, die sich die nationale nennt $\ll$.

Ottwalt, der zu ihr gehört hatte, schildert diese nationale Jugend, die im Kriege herangewachsen war und im Nachkrieg mit den Konsequenzen der Niederlage konfrontiert wurde. Es sind junge Frontoffiziere, die nichts gelernt hatten als das Waffenhandwerk, korporierte Studenten, denen die Inflation die materielle Basis entzog. Es ist kein nachträglich eingefärbter Schwarzweißbericht. Ottwalt weiß zu differenzieren. Sein erstes Buch ist kein hohes Kunstwerk - aber es hat viel von der Atmosphäre jener Jahre eingefangen, Wurzeln späteren Unheils bloßgelegt.

Als Ottwalt sein Buch beendete, 1928, waren im Glanze der "goldenen zwanziger Jahre diese Schatten der jüngsten Vergangenheit kaum noch bemerkbar. Die Wirren der Nachkriegszeit waren beinahe schon Geschichte, schienen Episode zu bleiben. "Eine neue Generation wächst heran, die von jenen unruhigen Zeiten kaum noch etwas weiß «, heißt es da im letzten Kapitel. Da waren noch keine zehn Jahre seit Kriegsende vergangen, und kaum fünf seit dem Hitlerputsch, mit dem dieses $Z$ wischenspiel endete. Aber es vergingen nach dem Erscheinen des Buches nur noch vier Jahre, bis das, was sich zwischen 1919 und 1923 in der Vorform gezeigt hatte, ausgewachsen war. Ahnungsvoll genug ist das letzte Kapitel des in Romanform gefaßten Erlebnisberichtes »Ruhe und Ordnung « überschrieben: „Und kein Ende....

Für die nach dem Zweiten Weltkrieg heranwachsende Generation ist auch das, was dann folgte, bereits Geschichte, vielfach noch im Dunkel gehaltene Geschichte. In der Kausalkette, die zur gegenwärtigen Geschichtssituation geführt hat, sind jene Kämpfe, Verstrickungen, Irrwege der ersten Nachkriegszeit Knotenpunkte; und manche Knoten, die damals geknüpft wurden, sind heute noch nicht aufgelöst. Ottwalts aufrichtiger - vor allem gegen sich selber schonungsloser - Erlebnisbericht ist aufschlußreich für die Täuschungen und Selbsttäuschungen der verlorenen Generation: ein Beitrag zur Zeitgeschichte, der der Vergessenheit entrissen werden sollte.

Dem Roman "Ruhe und Ordnung" folgte die "Geschichte des Nationalsozialismus«, eine aus den Quellen eigener Erfahrung mitgespeiste umfangreiche Analyse der Hitlerbewegung. Es ist der Versuch, das Phänomen des Erfolges dieser Bewe- 
gung nicht psychologisch, nicht vom "Führer « aus, sondern soziologisch zu deuten. $\mathrm{Daß}$ seine Deutung dreizehn Jahre später als Zeugnis vor einem internationalen Tribunal aufgerufen werden würde, konnte er so wenig ahnen, wie sein eigenes Schicksal: als Gefangener derer zu sterben, die er für seine Mitkämpfer hielt. Ende 193 I erschien Ottwalts zweiter Roman unter dem Titel »Denn sie wissen, was sie tun ". ${ }^{3}$ Es ist "Ein deutscher Justizroman". Ottwalt stellt ins Zentrum der Handlung eine erfundene Figur, genauer vielleicht eine synthetische Figur, den deutschen Richter der Zeit der Republik von Weimar. Er nennt ihn Friedrich Wilhelm Dickmann. Er macht aus ihm kein Schreckgespenst. Er stellt ihn als Menschen mit seinem Widerspruch dar, eher gutartig als überscharf, ausgestattet mit menschlicher Fehlbarkeit, Schwächen, aber im Grunde guten Willens, auf der Suche nach dem Recht in der Justiz. Womöglich hat Ottwalt, ohne es zu wissen, gerade durch diese Bemühung, nicht einseitig zu sehen, vom Klischee fernzubleiben, bei den Spitzenfunktionären der Partei sich mißliebig gemacht: er wurde des »Objektivismus « verdächtig, schien der "sozialistischen Parteilichkeit « zu ermangeln.

Dabei gibt er diesem Roman ein solides Fundament von Justizfällen der zwanziger Jahre, die manche Schreckensurteile der »Volks- und Sondergerichtshöfe " Hitlers, aber, wie Ottwalt an Leib und Leben erfuhr, auch der Judikatur Stalins vorwegnahmen. Alle diese in die Handlung eingewobenen Justizirrtümer oder Justizverbrechen sind dokumentarisch belegt. Der Autor mußte damals fürchten, daß manche dieser Fälle ehrbaren, rechtschaffenen Bürgern unglaubwürdig vorkommen würden, und er erbot sich, in jedem Einzelfalle den dokumentarischen Nachweis zu erbringen. Unterdessen wissen wir längst, daß der später ermordete Schriftsteller Felix Fechenbach mit strafprozessualer Willkür, Fälschung von Sachverständigengutachten, Vergewaltigung aller Rechtsnormen am 3. Oktober 1922 in München von einem Oberlandesgerichtsrat namens Hass wegen Veröffentlichung eines längst bekannten Telegramms zu elf Jahren Zuchthaus verurteilt wurde, während ein Oberleutnant Marloh, der ohne jede Notwendigkeit 29 friedlich zu einem Löhnungsappell erschienene Matrosen abknallen ließ, sich darauf ausreden durfte, er habe einen Befehl seiner vorgesetzten Dienststelle mißverstanden und freigesprochen wurde.

Der junge Referendar Dickmann will es nicht glauben, er »wehrt sich gegen diese Flut « von Nachweisen »wie ein Ertrinkender«. Er spricht von »böswilligen Entstellungen, von gemeinen Lügen, von gewissenloser Hetze. "In solchen Partien identifiziert sich Ottwalt mit seiner Figur; man spürt ergriffen seine Anteilnahme, man weiß auch, daß solche Ungerechtigkeiten, die ihm während seiner eigenen Studienzeit bekannt wurden, seine politische Entscheidung mit herbeigeführt haben. Und man folgert: wie ein Mann solchen unbeugsamen Rechtsbewußtseins dann später seelisch gelitten haben muß, nicht nur, weil eben dieses Unrecht, in vergröberter Form noch, ihn selber traf, sondern weil es, millionenfach, von denen begangen wurde, für die er im Namen der Gerechtigkeit optiert hatte, weil er vermeinte, sie würden gleich ihm dazu beitragen wollen, das Unrecht in der Welt zu mildern. Für seinen nicht positiven, aber keineswegs negativen Helden Dickmann weiß er Anteilnahme zu erwecken. Dickmann ist kein Streber, der über Leichen geht. Er sucht seinen Weg in einer Zeit, da das Fundament der Gesellschaft, die Rechtsstaatlichkeit, Erschütterungen ausgesetzt war. Er macht es sich nicht leicht. Er spricht sogar gelegentlich frei, wo andere Instanzen Zuchthaus verhängt haben. „Der Landgerichtsrat Dickmann schenkt sich nichts. Er besichtigt ein Arbeitshaus, um sich zu überzeugen, wie die Menschen, die er wegen Bettelns dort einweisen läßt, 
existieren. Er erleidet einen Nervenzusammenbruch. Am Ende beugt er sich. Die Verhältnisse sind stärker als er. Er ordnet sich ein. Demnächst wird er Landgerichtsdirektor werden, "Sein verstorbener Vater war es auch schon. « Man muß sich »nur erst durchringen. Jetzt kenne ich meinen Platz... .

Das Ende des Romans über das Justizunrecht beschwört fast visionär die Nächte der Gefangenen in ihren Zellen und die Einsamkeit ihrer Frauen. Es wird das Schicksal vorgezeichnet, das Ottwalt wenige Jahre später bis an sein Lebensende bestimmt war: in den Gefängnissen und Lagern des Landes, in dem er Zuflucht vor dem Terror des Nationalsozialismus gesucht hatte. Sein Weg führte ihn über Dänemark und eine umwegige Zwischenstation in Prag nach Moskau, seiner politischen Heimat.

Dort sind Plivier, Friedrich Wolf, Johannes R. Becher, Erich Weinert, Willi Bredel, Adam Scharer, Georg Schmückle seine Gefährten. Einige darunter nannten sich seine Freunde; sie waren die ersten, ihn zu verleugnen, und sie trugen nach dem Kriege die absurde Verleumdung, er sei ein Gestapo-Agent gewesen, nach Deutschland zurück. Seine Erzählung "Der Spitzel«, in der er die Verstrickung eines anderen deutschen Exilierten beschrieb, der, um sein Leben zu retten, einen Pakt mit dem Teufel, der Gestapo, geschlossen hatte, und dem dann später nach seiner Flucht in die Sowjetunion die Rechnung präsentiert wird - diese Erzählung, so verbreitete man, sei ein autobiographisches Geständnis seiner eigenen tragischen Schuld.

Erst ein Vierteljahrhundert später erfuhren wir durch das Buch seiner Frau, Waltraut Nicolas ("Viele tausend Tage «) die wahren Zusammenhänge und Quellen. Wir lernten daraus den Mann kennen, der das Modell der Erzählung gewesen war. Vieles erfuhren wir dann; auch dies, daß selbst die GPU die Beschuldigung der Spionage (geschweige denn die Unterstellung, Ottwalt sei ein Gestapo-Agent gewesen) nicht ernst nahm und nicht aufrechterhielt, denn weder Ottwalt noch seine Frau, die mit ihm zusammen verhaftet worden war, wurden zu den üblichen Strafen von pauschaliter 25 Jahren Gefängnis verurteilt, sondern nur routinemäßig wie Hunderttausende andere für befristete Zeit in ein Lager gesperrt - für Ottwalt allerdings wurde dann die Zeit unbefristet; er hat das Lager nicht überlebt. Damals, 1936, schreibt Waltraud Nicolas, verhaftete man zahlreiche Deutsche unter der Anschuldigung der Spionage.

"Man rechnete wohl so: wenn wir unter hundert einen wirklichen Spion fassen, hat sich die Sache gelohnt. Sie hatten gar kein Anklagematerial, behaupteten einfach: Du bist ein Spion, nun beweise mal das Gegenteil. Und wer einmal saß, kam nicht wieder heraus ... Wir kamen noch billig davon, nur fünf Jahre ...

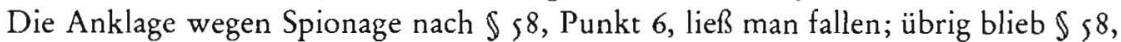
Punkt 10, "Agitation gegen den Staat «. In einer Novelle des Musikers, Dichters und Kammergerichtsrats E. T. A. Hoffmann wurde die Formel für solche Justiz bereits vor 1 go Jahren geprägt: "Den Täter haben wir. Die Tat werden wir noch finden.« Waltraut Nicolas, deren Größe es ist, daß sie nach allen bitteren Erfahrungen Menschen gerecht zu sehen und zu beurteilen vermag, berichtet in diesem Zusammenhang nicht nur von falschen Freunden, die Ottwalt nach seinem unverschuldeten Unglück nicht mehr kennen wollten, ihn verleugneten, ihn sogar verleumdeten, sondern auch von solchen, die sich später zu ihm bekannten und versuchten, ihm und seinem Werk Anerkennung zurückzugewinnen, der redliche Erich Weinert und seine resolute Frau Li unter diesen, vermutlich auch der noble Friedrich Wolf und die Brechts - die man nicht etwa verwechseln soll mit dem unter der Initiale "B« von Waltraut Nicolas mehrfach genannten früheren Freund von Ottwalt; dieser verräterische $\gg$ $B$ ist Becher. 
Bert Brecht und seine Frau Helene Weigel hingegen stehen in einem anderen Zusammenhang mit dem Schicksal von Ernst und Waltraut Ottwalt-Nicolas. Die beiden lebten im Frühjahr 1933 noch bedrängt, gefährdet, verfolgt und verlassen, halb illegal in Deutschland. Da erreichte eines Tages ein Brief aus der Schweiz Waltraut Nicolas:

"Ein Brief aus der Schweiz von einer unbekannten ,Tante Helene - wer ist das? rätselte ich. Ich kannte keine Tante Helene. Sonderbare Dinge schrieb sie: Die Krankheit der kleinen Erna mache ihr Sorge, aber hoffentlich könne Luftveränderung doch baldige Heilung bewirken. Bis dahin könnte gewiß ein schönes Buch dem Kind die Langeweile vertreiben. Es gäbe da ein so reizendes Kinderbuch von Karin Michaelis: die Grüne Insel. Das würde Erna gewiß Freude machen ...

Solche Tantenbriefe mußte man zu deuten wissen. Daß mit der kranken kleinen Erna der gefährdete Ernst Ottwalt gemeint war, verstand sich; und daß die Tante Helene niemand anderer als Helene Weigel-Brecht sein konnte, war zu vermuten. Frau Waltraut kaufte das Kinderbuch von Karin Michaelis; es war illustriert mit einer bunten Landkarte der dänischen Insel Thurö. Und nach ihrer daraufhin unternommenen Flucht verlebten Ottwalts auf Thurö am Sund als Gäste von Karin Michaelis gemeinsam mit Brechts ihre »letzte glückliche Zeit«.

"Dort war Zuflucht vor der Gestapo, dort dachten wir noch nicht an die NKWD. Vier Jahre später, als wir im vergitterten Wagenabteil durch Rußland fuhren und uns fragten: wo möchten wir sein, wenn wir frei wären? sagten wir beide: in Dänemark.«

Am Vorabend des 7. November 1936, dem Jahrestag der sowjetischen Revolution, wurden die Ottwalts bei einem Spaziergang auf dem Roten Platz von der Straße weg verhaftet. Waltraud Nicolas hat in ihrem Buch "Die Kraft, das Ärgste zu ertragen" die Stationen des Passionsweges, der für Ernst Ottwalt irgendwann irgendwo in einem Lager am Eismeer, für sie mit ihrer Auslieferung nach Nazideutschland endete, genau und eindringlich beschrieben. Die Tragödie begann als Farce. Bei dem abendlichen Spaziergang über den Roten Platz in Moskau tritt ein Fremder auf die beiden zu und behauptet, Ottwalt sähe einem Mann ähnlich, der ihm, dem Nachfragenden, in einem Sanatorium auf der Krim seine Uhr und seine Dokumente entwendet habe. Ottwalts waren nie auf der Krim gewesen; sie beschieden den jungen Mann lächelnd, daß da eine Verwechslung vorliege. Der entschuldigte sich verlegen - aber, wenn es den Herrschaften nichts ausmache, so könne man den Fall sogleich klarstellen; nebenan sei die Polizeiwache, wo sie sich ausweisen könnten. Ottwalt lachte: "Das erste Mal im Leben, daß man mich für einen Uhrendieb hält warum sollen wir uns den Witz entgehen lassen? « Sie gingen mit dem jungen Mann auf die Wache. Als sie, noch lächelnd über dieses absurde Abenteuer, die Tür hinter sich schließen, ist für Ernst Ottwalt die letzte Minute seines Lebens in - relativer Freiheit vergangen.

Danach war vom »Uhrendiebstahl« nie mehr die Rede, hingegen von "Spionage ", von »Trotzkismus«, »Agitation gegen den Staat«; eines wie das andere so frei erfunden wie der Aufhänger dieser Verhaftung, der Uhrendiebstahl auf der Krim, wo die Ottwalts nie gewesen waren. Das geht durch Tage, Wochen, Monate, Jahre, durch Gefängnisse, Einzelzellen, Massenzellen, Baracken, Lager, Transporte, Rücktransporte, neue Einzelzellen, neue Massenzellen, neue Lager, Straflager, Arbeitslager; einmal noch kreuzen sich die Wege von Ernst und Waltraut Ottwalt-Nicolas: auf einem Gefangenentransport in einem Gefängnisauto, das dort nicht "grüne Minna«, sondern »schwarzer Rabe« genannt wird. Waltraut Nicolas hat in ihrem Buch »Die Kraft, das Ärgste zu ertragen« dieses Wiedersehen geschildert:

"Dicht neben meinem Ohr höre ich plötzlich wieder diese flüsternde Stimme: 
Schau her, aber gib acht, daß die Konvois (Wachtposten) nichts merken. $<$ Vorsichtig wende ich den Kopf nach der Richtung, aus der die Stimme kam. Da sehe ich neben mir hinter einem Gitter bleiche Männergesichter sich aus dem Dunkel herausschälen. Mager und ausgehöhlt sind sie, von struppigen Bärten umrahmt; aus tiefen Schattenhöhlen starren leuchtende Augen auf mich. Und eins dieser graublassen, bärtigen Gesichter, das von einem unbeschreiblichen Lächeln erhellt ist, erkenne ich. 'Du - du bist noch da? flüstere ich. , Ja, ich bin noch da! sagt er mit einer Betonung, die vielmehr ausdrückt: mich kann man einfach auslöschen...

Wie schmal sein Gesicht geworden ist, wie schrecklich verändert! Tiefe Falten haben es durchzogen, das Haar über der hohen Stirn ist weiß geworden. Ein alter Mann und er ist erst 36 Jahre alt! Aber das Lächeln, das nur den einen Mundwinkel verzieht, wenn ihn etwas innerlich anrührt - das ist dasselbe geblieben wie früher...

,Bist du gesund? fragt er leise.

Jar, lüge ich, 'ganz gesund! Und du?

,Ich auch $<$, lächelt er, aber die tiefen Schatten unter seinen Augen sagen das Gegenteil. , Wie war die Untersuchung?c

,Punkt sechs, zehn und elf^, flüstere ich bedrückt.

'Scher dich nicht darum, das ist ja alles Theater, lacht er. Sie verhaften Deutsche; aber zum Verhör haben sie mich nur ein einziges Mal geholt und nicht einmal Protokoll geführt.؛

Mir wird ganz leicht ums Herz. Nur ein Verhör und ohne Protokoll - sie werden ihn nicht erschießen...!

Der Zufall will es, daß sie in dem Transportzug, der die Gefangenen nach Stalingrad bringt, im selben Abteil zusammenbleiben. Zwei Tage konnten sie sich einander mitteilen:

"... und diese Tage werden nachklingen durch Jahre, die uns vielleicht noch voneinander trennen werden. Drei, fünf, zehn Jahre? Wir wissen es nicht...

Im Gefängnis von Stalingrad sahen sie einander zum letztenmal. Dann führte sie ihr Weg für immer auseinander.

Siebentausendachthundertundzwanzig Tage und Nächte hoffte Waltraut Nicolas, daß Ernst Ottwalt noch am Leben sei, irgendwo in einem der Lager Stalins noch am Leben. Sie stand wie so viele andere Frauen und Väter und Mütter im Heimkehrerlager Friedland und hob die Tafel hoch, auf der geschrieben war:

Ernst Gottwalt Nicolas

Ernst Ottwalt

Geb. I3. II. I90I

Letzte Nachricht Herbst 1940

Kuloi-Lag, Sjelo Talagi, Gorod Archangelsk.

Alfred Kantorowicz ${ }^{4}$

\footnotetext{
4 Beı dem Beıtrag handelt es sıch um die gerıngfügıg gekürzte Fassung des Porträts Ernst Ottwalt, aus: Alfred Kantorowicz, Deutsche Schicksale, Intellektuelle unter Hitler und Stalin, Europa Verlag, Wien 1964 S. 171 ff. (Red. KJ).
} 


\title{
Entscheidungen
}

\section{Beschluß des Amtsgerichts Berlin-Charlottenburg vom 9. I2. I975}

\author{
Beschluß \\ in der Wohnungseigentumssache Pariser Straße [...], (Grundbuch Berlin [. . .]), \\ Antragstellerin: Die Boden-Finanz Gesellschaft für Grundstücksverwertung mbH \\ und Immobiliengeschäfte $\mathrm{KG}$, \\ Beteiligte: \\ I. die Verwalterin Wirtschaftsgenossenschaft Berliner Grundbesitzer, Berlin \\ II. die Wohnungseigentümer der Wohnungen [. . . ] \\ wird beschlossen: \\ I. Der Beschluß der Wohnungseigentümerversammlung vom 29.3. 75 zu Punkt 5) \\ der Tagesordnung betreffend Vermietung eines Ladens der Antragstellerin an den \\ Club Pablo Neruda, wird insoweit für unwirksam erklärt, als der Antragstellerin \\ aufgegeben worden ist, eine Räumung binnen Monatsfrist zu verlangen. \\ 2. Im übrigen wird der Antrag zurückgewiesen. \\ 3. Die Gerichtskosten des Verfahrens hat die Antragstellerin zu tragen. Außerge- \\ richtliche Kosten werden nicht erstattet. \\ 4. Der Geschäftswert wird auf 7200,- DM festgesetzt.
}

\section{Gründe}

Die Antragstellerin ist die Eigentümerin der Wohnungen bzw. Läden Nr. I, 2, 3 und 6 der Anlage. Sie hat im Dezember 1974 einen ihrer Läden an den »Club Pablo Neruda übergeben, hat der Verwalterin die Vermietung dieses Ladens an diesen Club mitgeteilt und hat um Genehmigung gebeten. In der Teilungserklärung ist nämlich bestimmt, daß nur mit Genehmigung des Verwalters vermietet werden kann und daß der Verwalter diese Genehmigung nur aus wichtigem Grund versagen darf. Die Verwalterin antwortete im gleichen Monat, sie würde den Fall der Wohnungseigentümerversammlung vorlegen.

In der Wohnungseigentümerversammlung vom 29. 5 . 75 wurde hierüber zu Punkt 8 der Tagesordnung verhandelt. Im Protokoll heißt es:

"Die anwesenden Wohnungseigentümer beauftragen mehrheitlich, die nach $\$ 6$ Absatz 2 der Teilungserklärung erforderliche Verwalterzustimmung zur Vermietung eines Ladens der Boden-Finanz GmbH \& Co, KG an den Club Pablo Neruda aus wichtigem Grund zu verweigern, da begründete Befürchtung besteht, daß die Mieter sich in die Hausgemeinschaft nicht einfügen werden, Lärm- und Schmutzbelästigung bereits aufgetreten ist und sich einzelne Eigentümer durch die Mieter bedroht fühlen. Die Boden-Finanz GmbH \& Co. KG wird zugleich aufgefordert, vom Mieter die sofortige Räumung des Ladens ohne Einhaltung einer Kündigungsfrist zu verlangen. Räumungsfrist: ein Monat nach Zugang dieses Protokolls bei der Boden-Finanz GmbH \& Co KG.

Hierfür stimmen 5732/10 00ostel Miteigentumsanteile; der Stimme enthalten haben 